\title{
Biochemical changes of intervertebral discs in patients with spondylolisthesis or with tears of the posterior annulus fibrosus
}

\author{
SALLY ROBERTS, ${ }^{1}$ HELEN K. BEARD, ${ }^{1}$ AND JOHN P. O'BRIEN ${ }^{2}$ \\ From the ${ }^{1}$ Charles Salt Research Centre and the ${ }^{2}$ Department for Spinal Disorders, Robert Jones and Agnes \\ Hunt Orthopaedic Hospital, Oswestry, Shropshire SY10 7 AG
}

SUMMARY Surgically removed discs from patients with either spondylolisthesis or tears of the posterior annulus fibrosus were analysed for water, proteoglycan, and collagen content and compared with post-mortem control material. Discs from patients with spondylolisthesis had a reduced proteoglycan content in all sections sampled and less collagen in the outer annular layers. In contrast discs containing tears in the posterior annulus were unaltered biochemically, although extended studies on 2 patients indicated that there may be localised biochemical changes in the region of the tear itself. Collagen types I, II, and III and proteoglycan distributions were studied qualitatively by immunofluorescence. Collagen types I and III appeared to be reduced in discs from patients with spondylolisthesis, but again little change was found in patients with tears in the posterior annulus fibrosus.

Spondylolisthesis describes the forward displacement of a vertebral body, and is commonly seen at the lumbosacral joint. True spondylolisthesis occurs in approximately $6 \%$ of the population, ${ }^{1}$ and in the vast majority of cases the displacement is slight. There is, however, a distinct group, usually seen in adolescents, where gross displacement occurs with the body of L5 coming to lie almost in front of the first sacral body. ${ }^{1}$

Traumatic injuries of the lumbar spine frequently result in tears of the annulus fibrosus which can be detected by discography. ${ }^{2}$ Such tears commonly occur at the lumbosacral joint, are located posteriorly, and are clearly visible during subsequent surgery for disc removal by the anterior approach. These groups of patients, both those with spondylolisthesis, either mild or severe, and those with tears in the posterior annulus represent clinical conditions in which the intervertebral discs have been subjected to abnormal stress or trauma. There is evidence from $x$-rays and discograms for disc degeneration in all these patients, but no biochemical or morphological studies have been done on either group.

The aim of this study has been to investigate biochemical changes in sections of anterior annulus fibrosus and nucleus from the appropriate intervertebral discs removed at surgery. Collagen,

Accepted for publication 19 January 1981.

Correspondence to Dr S. Roberts. proteoglycan, and water profiles were obtained, together with qualitative distribution patterns of collagen types I, II, and III and compared with age-matched post-mortem controls.

\section{Materials and methods}

COLLECTION OF MATERIAL

Specimens of intervertebral disc were taken from patients undergoing anterior disc excision and spinal fusion. Diagnosis was made on clinical and radiological evidence. Three patients underwent fusion for mild spondylolisthesis, while 6 severe cases had both reduction and fusion. ${ }^{3}$ Ten patients had tears of the posterior annulus fibrosus. ${ }^{2}$ One patient case (4) had tears in 2 discs, another (case 9) had a posterior tear in one disc and a mild degree of spondylolisthesis in another. The age range for spondylolisthesis patients was 9 to 39 years, the 6 severe cases being under 21 . All affected discs were lumbosacral. Patients with tears of the posterior annulus ranged in age from 24 to 40 years; 8 were lumbosacral and 3 were lumbar 4-5 in position.

At surgery the sagittal anterior section of disc was removed. It contained outer and inner annulus, usually some nuclear material, and in 2 cases of annular tears (Cases 1 and 8 ) included tissue adjacent to the tear. Immediately on removal the tissue was 
placed in a sterilised chamber kept at $100 \%$ humidity to prevent evaporation. ${ }^{4}$ For long-term storage the specimen was frozen in $\mathrm{N}$-hexane (boiling range $67-70^{\circ} \mathrm{C}$, BDH Chemicals Ltd) at $90^{\circ} \mathrm{C}$ and kept in liquid nitrogen.

Control material from post-mortem spines obtained within 24 hours of death was treated in a similar manner. The same area was removed from corresponding intervertebral discs of age-matched and where possible sex-matched subjects.

\section{WATER CONTENT}

Disc material was removed from storage, divided radially into approximately $3 \mathrm{~mm}$ sections, and labelled from $\mathrm{A}$, the outer anterior annulus, across the disc as far as the tissue allowed. The average extended to $E$ but in the case of 2 controls, where there were complete sections of intervertebral disc, as far as $J$ at the outer posterior annulus. This was to correspond with the 2 annular tear patients in whom there was extensive excised material, including some of the tear region. The wet weight of each section was obtained and the dry weights measured after drying to a constant weight over phosphorous pentoxide. Water content was calculated as \% water/wet weight.

\section{COLLAGEN CONTENT}

Dried sections of disc (approximately $5 \mathrm{mg}$ ) were accurately weighed and hydrolysed in $6 \mathrm{M} \mathrm{HCl}$ for 16-24 hours at $100^{\circ} \mathrm{C}$. After cooling, the solutions were neutralised to $\mathrm{pH} 7 \cdot 0$ with $5 \mathrm{M} \mathrm{NaOH}$, with BDH 678 used as indicator. Approximately $0.5 \mathrm{~g}$ of a mixture of charcoal and Dowex 1-X8 $(1: 2 \mathrm{w} / \mathrm{w})$ was added to remove charred carbohydrate and decolourise the solution. Samples were filtered and made up to $10 \mathrm{ml}$ with distilled water. Dilutions of $80 \mu \mathrm{l}$ in $2 \mathrm{ml}$ distilled water were made, and the hydroxyproline content was estimated colourimetrically according to Woessner. ${ }^{5}$ The percentage collagen/wet weight for each section was calculated from mean hydroxyproline values (of at least 6 readings, mean SD 6.9) with a multiplication factor of $7 \cdot 55$, based on $13 \cdot 2 \% \mathrm{w} / \mathrm{w}$ hydroxyproline for type I collagen, which is the major collagen found in the anterior annulus. ${ }^{6}$

\section{PROTEOGLYCAN CONTENT}

Accurately weighed dried sections (approximately $5 \mathrm{mg}$ ) of disc were hydrolysed with 9 units of papain (type IV; Sigma) in $1 \mathrm{ml}$ of $0.01 \mathrm{M}$ cysteine- $\mathrm{HCl} /$ $0.005 \mathrm{M}$ EDTA $/ 0.2 \mathrm{~N} \mathrm{Na}$ acetate, $\mathrm{pH} 6.0$, at $60^{\circ} \mathrm{C}$ for 3-6 hours until all the tissue went into solution. Hydrolysed samples were then made up to $10 \mathrm{ml}$ volume with saturated benzoic acid solution and aliquots analysed for uronic acid. ${ }^{7}$ The proteoglycan content was calculated from mean uronic acid values (of at least 4 readings, mean SD 7.4) with a multiplication factor of $7 \cdot 00.8$

Significance between means was calculated according to Student's $t$ test for a small sample number and where the 2 means are not assumed to have the same variance.

\section{IMMUNOCHEMICAL LOCALISATION OF}

COLLAGEN TYPES AND PROTEOGLYCAN

The preparation and characterisation of antibodies to collagen types I and II and proteoglycan has been described previously. ${ }^{9}$ Antisera to collagen types I and II were specific for native collagen molecules. Antisera to proteoglycan showed greatest reaction with the hyaluronate binding region of cartilage and disc proteoglycan, together with weak reaction to the keratan sulphate and chondroitin sulphate richregions. Type III collagen was prepared from human placenta and used to raise antisera as described previously. ${ }^{10}$

Nine discs from patients with annular tears and 6 with severe spondylolisthesis were investigated by immunofluorescence and compared with 6 and 5 controls, respectively. Disc tissue, stored under liquid nitrogen, was sectioned on a Bright's rotary cryostat. Sections $(7 \mu \mathrm{m})$ were stained for collagen by indirect immunofluorescence as previously described ${ }^{9}$ after being treated with $0 \cdot 1 \mathrm{mg} \mathrm{ml}^{-1}$ trypsin (type III, 11700 units $\mathrm{mg}^{-1}$ protein, Sigma) for $15 \mathrm{~min}$ at $37^{\circ} \mathrm{C}$. This enhanced the staining of collagen, possibly by removing other proteins ${ }^{11}$ or proteoglycan, ${ }^{12}$ which were covering the fibres. It did not appear to release any type III molecules from the tissue, since less than $0.2 \%$ of the tissue hydroxyproline was lost after protease incubation. Sections stained with antiproteoglycan or haemotoxylin and eosin were not pretreated with trypsin. All sections were fixed in freshly prepared $4 \%$ paraformaldehyde

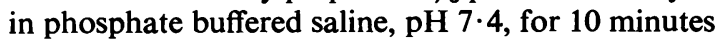
at room temperature before being treated with antisera. Fluorescence was viewed on a Leitz Dialux 20 microscope fitted with incident illumination and Phloempak 2.4 filter unit.

\section{Results}

WATER CONTENT

Percentage of water content was measured in 96 sections of patient material and 74 controls. The mean values for both patient groups were compared with those for the corresponding control group at each site across the intervertebral disc. In all cases the percentage water/wet weight increased from outer to inner annulus, as has been shown in canine discs. ${ }^{13}$ No significant differences were found when comparing patient and control groups (Figs. 1a, 1b). 

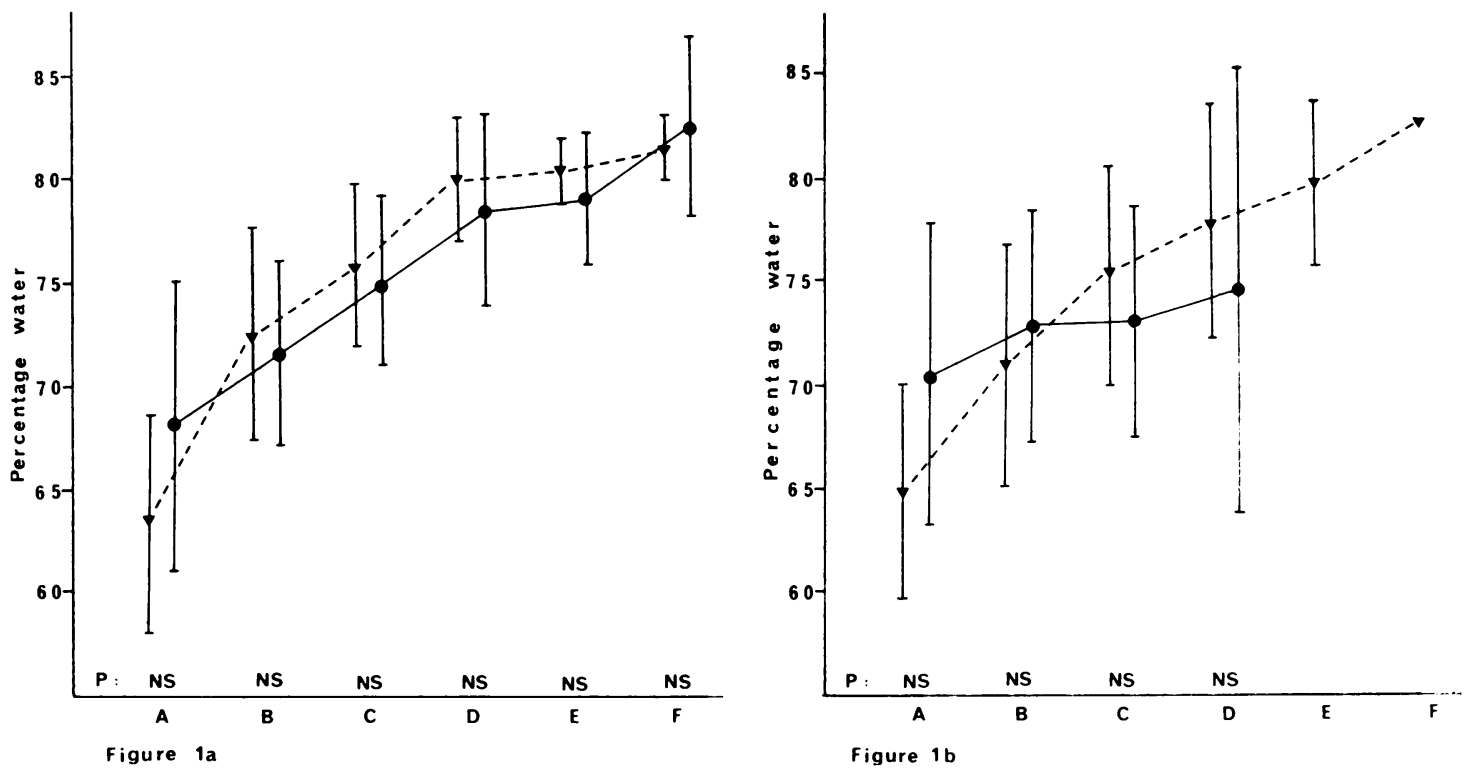

Fig. 1 Variation of water content across disc. (a) Posterior annular tear patients. (b) Spondylolisthesis patients. Patients. $\nabla---\nabla$ Controls. Vertical bars show standard deviation.

\section{COLLAGEN CONTENT}

The collagen content for all groups was found to decline from outer to inner annulus, and again this is consistent with previous work. ${ }^{14}$ The annular tear patients gave values for percentage collagen/wet weight closely resembling those obtained for theo control group (Fig. 2a). Spondylolisthesis patients were also similar to their controls, with the exception of section $\mathrm{A}$, which had significantly less collagen than the controls (Fig. 2b).

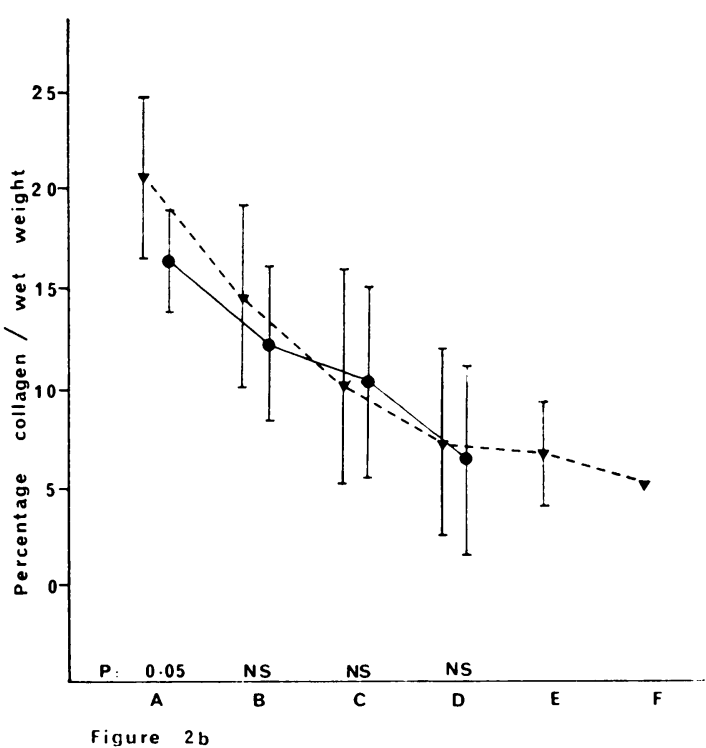

Fig. 2 Variation of percentage collagen/wet weight across disc. (a) Posterior annular tear patients. (b) Spontylolisthesis patients.

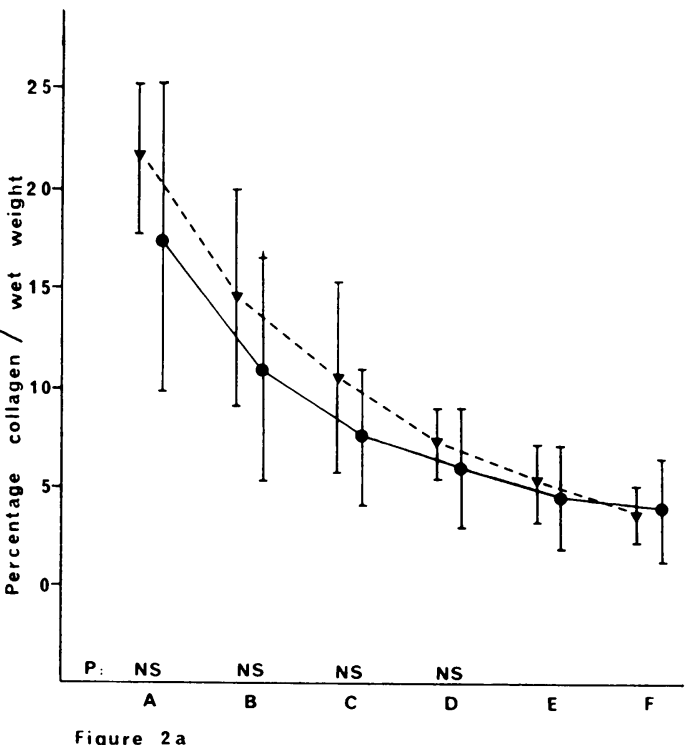

(a) Posterior annular tear 

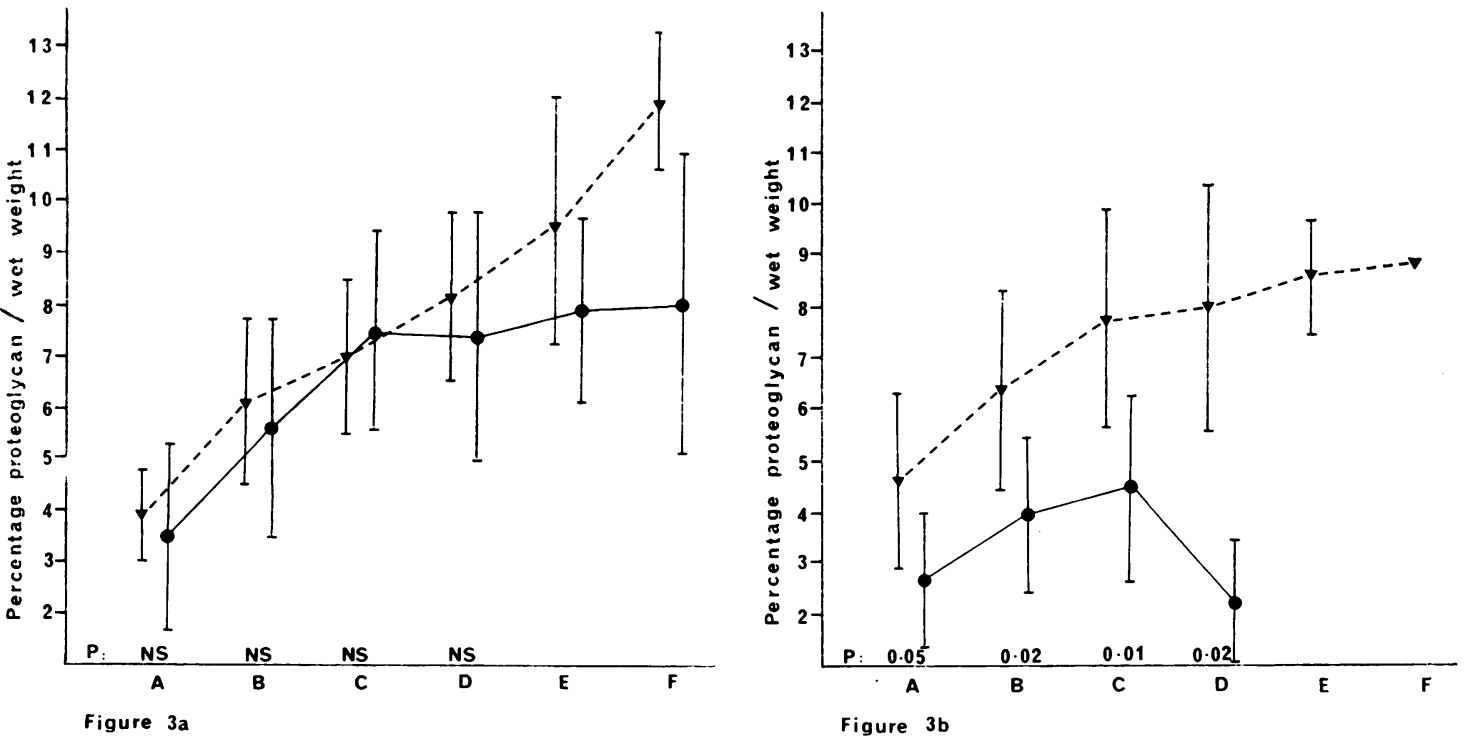

Fig. 3 Variation of percentage proteoglycan/wet weight across disc. (a) Posterior annular tear patients.

(b) Spondylolisthesis patients. $\longrightarrow$ Patients. $\nabla--\nabla$ Controls. Vertical bars show standard deviation.

\section{PROTEOGLYCAN CONTENT}

The proteoglycan content increased from outer to inner annulus. Again there was no significant difference between corresponding sections from discs with annular tear and controls, though there was an apparent lowering of percentage proteoglycan/wet weight in patient material in the nucleus and therefore towards the region of the tear (Fig. 3a). The spondylolisthesis group, however, gave significantly lower values in all sections analysed (Fig. 3b). The percentage loss compared with that in controls is shown in Table 1. In one patient (Case 9), who had one disc with an annular tear and another with spondylolisthesis, the percentage of loss of proteoglycan was calculated for each (Table 2). As expected the disc with a spondylolisthesis showed a greater loss in proteoglycan in all but the outer section of annulus.

Complete profiles were done on 2 patients (Cases 1 and 8) in which posterior tears of the annulus were clearly visible at operation. Results for percentage water, collagen, and proteoglycan content/wet

Table 1 Percentage proteoglycan lost in spondylolisthesis discs

\begin{tabular}{ll}
\hline Section of disc & $\%$ Loss \\
\hline A & $43 \cdot 5$ \\
B & $39 \cdot 1$ \\
D & $43 \cdot 6$ \\
\hline
\end{tabular}

Table 2 Comparison of percentage of proteoglycan loss in patient (Case 9) between disc with spondylolisthesis (1) and disc with posterior annular tear (2)

\begin{tabular}{lll}
\hline Section of disc & $\%$ loss in 1 & $\%$ Loss in 2 \\
\hline A & $68 \cdot 3$ & $70 \cdot 5$ \\
B & $58 \cdot 9$ & $42 \cdot 3$ \\
D & $73 \cdot 5$ & $44 \cdot 3$ \\
\hline
\end{tabular}

weight were compared with their respective agematched controls (Figs. 4a, 4b). These results suggested that in one patient (Case 1) there may have been changes, notably loss of collagen and increase of proteoglycan and water, which occurred around the tear and were not apparent in the remainder of the disc. The other patient resembled the controls. There was some difficulty in matching the positions of patient and control exactly, because the complete disc was removed in the case of the post-mortem material while in the patient it was obviously limited by the danger of damage to the dura. The tear region was estimated to correspond with section I of the controls (Fig. 4).

\section{IMMUNOCHEMICAL LOCALISATION OF} COLLAGEN TYPES AND PROTEOGLYCANS

The staining patterns in all the control material showed a distribution pattern comparable to that in pig and normal human intervertebral discs. ${ }^{10}$ The 
Figure 4 a

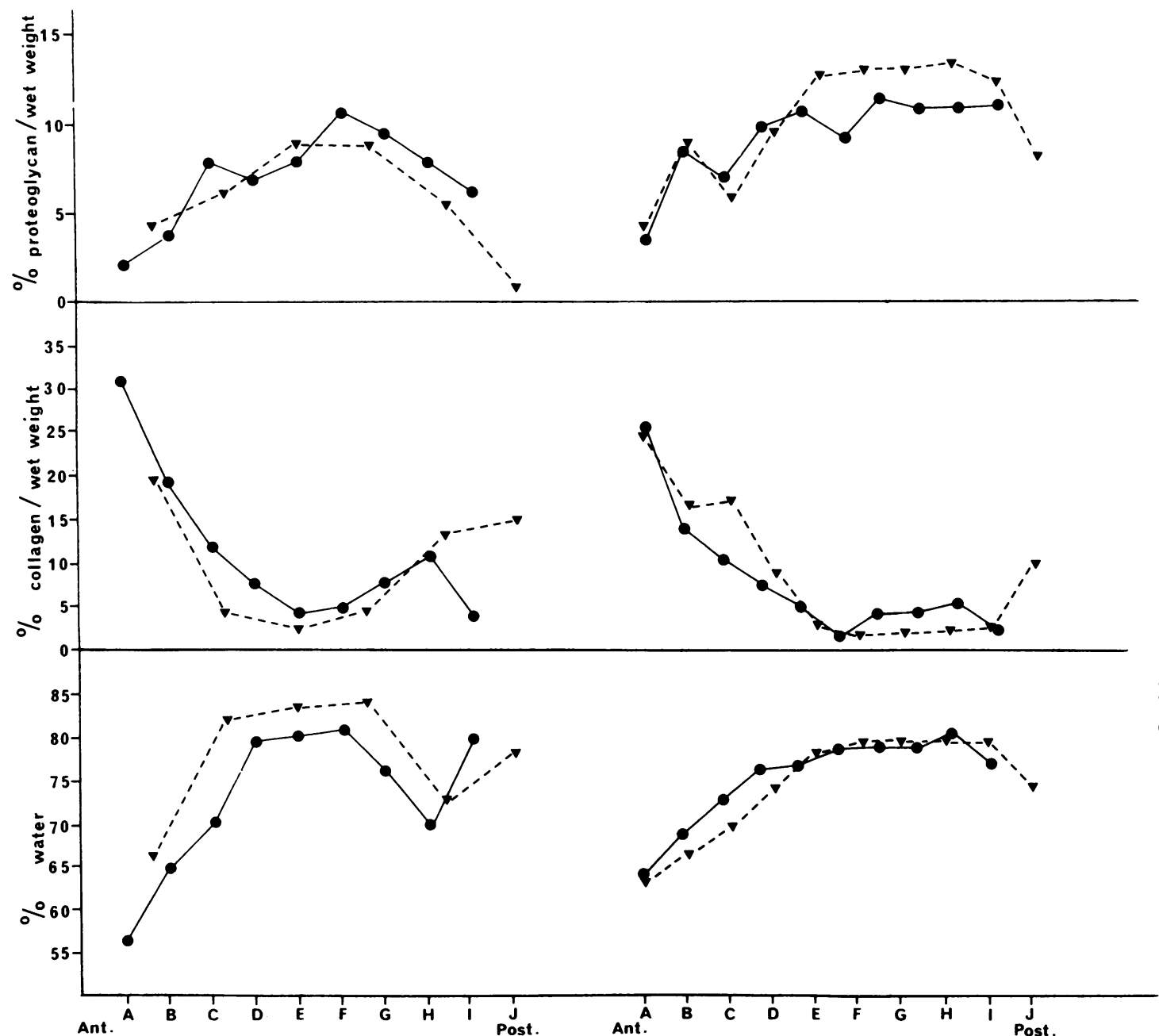

Fig. 4 Variation in water, collagen, and proteoglycan content across the discs in 2 posterior annular tear patients. $I$ is the tear region. (a)

results are summarised in Table 3. Staining with type I antisera was generally strongest in the outer annulus, while that for type II was stronger in the inner annulus and nucleus. Antiproteoglycan gave a similar pattern with the nucleus staining strongly, but no staining in the outer annulus. Antisera to type III, however, gave a discrete pericellular pattern, occurring mainly in the nucleus and inner annulus. Chondrocytes and fibroblasts both gave pericellular staining. Discs from patients with annular fissures showed little deviation from this pattern except in 2 patients, Cases 1 and 4 (L5-S1), which showed relatively weak and infrequent pericellular staining with antisera to type III.
In the group of spondylolisthesis patients there appeared to be a greater difference from the control material. Some discs showed weaker staining to anti-type I collagen, while most showed fewer cells and weaker staining with anti-type III. One patient (Case 17) showed particularly weak staining with antisera to all 3 types. When the method was repeated for this patient after pretreating the frozen sections with chondroitinase ABC (Miles Biochemicals; 5 units $\mathrm{ml}^{-1}$ ) for $30 \mathrm{~min}$ at $37^{\circ} \mathrm{C}$, there was markedly enhanced staining for all 3 types. This did not occur with post-mortem disc material and suggests that proteoglycan may have masked the collagen fibres in this specimen. 
Table 3 Fluorescent staining intensities with antisera to collagen types I, II, and III and proteoglycan

\begin{tabular}{|c|c|c|c|c|c|c|c|c|}
\hline Patient & Sex & Disc & Age & $\begin{array}{l}\text { Age of } \\
\text { onset/trauma }\end{array}$ & $I$ & II & $I I I$ & $P g$ \\
\hline \multicolumn{9}{|c|}{ Posterior annular tears } \\
\hline 1 & $\mathbf{F}$ & L5-S1 & 24 & 12 & + & \pm & + Few & ++ \\
\hline 2 & $\mathbf{M}$ & L5-S1 & 24 & 23 & + & ++ & ++ & $+t+$ \\
\hline 3 & $\mathbf{F}$ & L5-S1 & 26 & 22 & + & + & +++ & +++ \\
\hline 4 & $\mathbf{M}$ & $\begin{array}{l}\text { L4-5 } \\
\text { L5-S1 }\end{array}$ & 32 & & $\begin{array}{l}+ \\
+\end{array}$ & $\begin{array}{l}+ \\
+\end{array}$ & $\begin{array}{l}++ \\
+ \text { Few M }\end{array}$ & $\begin{array}{l}+++ \\
++\end{array}$ \\
\hline 5 & $\mathbf{F}$ & L5-S1 & 38 & 30 & + & + & ++ & NT \\
\hline 6 & $\mathbf{M}$ & L5-S1 & 38 & 30 & + & + & ++ & $+t+$ \\
\hline 7 & $\mathbf{M}$ & L5-S1 & 38 & 31 & NT & + & ++ & ++ \\
\hline 8 & $\mathbf{F}$ & L4-5 & 40 & 33 & + & + & $+++\mathbf{M}$ & +++ \\
\hline 9 & $\mathbf{M}$ & L4-5 & 31 & 26 & NT & NT & NT & NT \\
\hline 10 & $\mathbf{M}$ & L5-S1 & 37 & 31 & NT & NT & NT & NT \\
\hline \multicolumn{9}{|c|}{ Controls } \\
\hline 11 & $\mathbf{F}$ & L5-S1 & 23 & & \pm & $+t$ & +++ & $+t+$ \\
\hline 12 & $\mathbf{M}$ & L5-S1 & 23 & & + & + & ++ & $++t$ \\
\hline 13 & $\mathbf{M}$ & $\begin{array}{l}\text { L4-5 } \\
\text { L5-S1 }\end{array}$ & 28 & & $\begin{array}{l}++ \\
+\end{array}$ & $\begin{array}{l}++ \\
++\end{array}$ & $\begin{array}{l}+++ \\
+++\end{array}$ & $\begin{array}{l}++ \\
++\end{array}$ \\
\hline 14 & $\mathbf{M}$ & L5-S1 & 40 & & + & + & ++ & $+t$ \\
\hline 15 & $\mathbf{F}$ & L5-S1 & 42 & & NT & ++ & $++t$ & $+t+$ \\
\hline 16 & $\mathbf{M}$ & L5-S1 & 38 & & NT & NT & NT & NT \\
\hline \multicolumn{9}{|c|}{ Spondylolisthesis } \\
\hline 17 & F & L5-S1 & 9 & 7 & \pm & \pm & $\pm \mathbf{M}$ & $t+$ \\
\hline 18 & $\mathbf{F}$ & L5-S1 & 13 & 10 & \pm & $\overline{ \pm}$ & + Few & + \\
\hline 19 & $\mathbf{M}$ & L5-S1 & 14 & 13 & $\overline{+}$ & $\overline{+}$ & + Few & ++ \\
\hline 20 & $\mathbf{M}$ & L5-S1 & 16 & 15 & \pm & \pm & ++ & $++t$ \\
\hline 21 & $\mathbf{M}$ & L5-S1 & 17 & 15 & ++ & + & + Few $\mathbf{M}$ & ++ \\
\hline 22 & $\mathbf{M}$ & L5-S1 & 21 & 17 & + & + & + Few & ++ \\
\hline 23 & $\mathbf{M}$ & L5-S1 & 16 & 13 & NT & NT & NT & NT \\
\hline 9 & $\mathbf{M}$ & L5-S1 & 31 & 26 & $\mathrm{NT}$ & NT & NT & NT \\
\hline 24 & $\mathbf{M}$ & L5-S1 & 39 & 38 & NT & NT & NT & NT \\
\hline \multicolumn{9}{|c|}{ Controls } \\
\hline 25 & $\mathbf{M}$ & L5-S1 & 9 & & + & + & $++\mathbf{F e w}$ & ++ \\
\hline 26 & $\mathbf{F}$ & L4-5 & 12 & & + & + & $++t$ & ++ \\
\hline & & L5-S1 & 12 & & ++ & + & ++ & $+t+$ \\
\hline 27 & $\mathbf{M}$ & L5-S1 & 15 & & ++ & \pm & ++ & ++ \\
\hline 28 & $\mathbf{M}$ & L5-S1 & 21 & & + & + & $t+$ & ++ \\
\hline
\end{tabular}

$\mathbf{M}=$ matrix staining. $\mathbf{P g}=$ proteoglycan. Few $=$ fewer cells staining. NT $=$ not tested.

\section{Discussion}

The annular tear patients gave no significant changes from normal in the biochemical studies, but since only the anterior annulus and sometimes the nucleus were sampled in all but 2 cases this was not surprising, for the pathology appears to be in the posterior part of the disc. The finding that the anterior regions of the disc were biochemically unchanged fits with clinical evidence and observations at surgery. In 1 of the 2 patients (case 1) from whom more material was obtained there was a suggestion that biochemical changes might be taking place in the posterior annulus. The increased water and proteoglycan observed together with reduced collagen were not in accordance with findings in other disc pathology. ${ }^{15-17}$ However, studies in animals suggested that the biochemical changes following trauma may be highly variable. Lipson and Muir ${ }^{18}$ induced a rabbit model of disc degeneration which showed that the water content initially fell and then rose rapidly again, before showing a gradual loss over an extended period. Proteoglycan levels followed this closely. If there is a similar trend after trauma in annular tear patients the water content in relation to normal would depend on time of sampling, and could explain the apparent discrepancies of water and proteoglycan levels.

There was a significant loss of proteoglycan in the group of spondylolisthesis patients, whether mild or severe and in all sections sampled. These changes were similar to those found in osteoarthritic articular cartilage, which had lower proteoglycan, ${ }^{19}$ accompanied by increased water ${ }^{20} 21$ and an increase in collagen synthesis and chondrocyte activity ${ }^{22}$ In this situation it appeared that the chondrocytes themselves were affected and either had a decreased ability to synthesise proteoglycan ${ }^{19}$ or synthesised proteoglycans which were more readily extracted, ${ }^{23}$ possibly owing to shorter chondroitin sulphate chains. ${ }^{2325}$ The mechanical stress experienced in the disc in spondylolisthesis and in articular cartilage in osteoarthritis may be similar in inducing biochemical changes in the proteoglycans in these tissues.

Collagen levels in degenerative joint disease showed less dramatic changes; indeed in many studies they appeared not to be altered at all. ${ }^{21}{ }^{26}$ In this study there was a significant change only in the outer annular section of the spondylolisthesis group, which was lower than the controls. Venn and Maroudas $^{20}$ found a similar loss in osteoarthritic cartilage. There is some evidence for degraded collagen fibrils arranged in a halo round chondrocytes of prolapsed discs, ${ }^{27}$ and of 'reaction products' round chondrocytes in normal and more intensely in chondromalacic cartilage. ${ }^{28}$ In other studies both collagen synthesis and degradation were increased in osteoarthritic cartilage, ${ }^{22}$ so that an overall balance was maintained.

The apparent decrease in type I and type III collagen in spondylolisthesis discs was interesting. No corresponding rise in type II collagen was seen, but the distribution of other collagen types (for examples IV and V) was not investigated. Since the technique used was entirely qualitative and dependent on the tissue architecture, the changes observed may have resulted from the redistribution of other tissue components such as proteoglycan rather than a quantitative change in collagen type ratio. However, changes in type of collagen have been demonstrated in osteoarthritic cartilage, where type I was synthesised as well as type II, ${ }^{29}{ }^{30}$ but this was refuted by Floman et $a .^{31}$ and Lippiello et al. ${ }^{22}$ Adam et al. ${ }^{32}$ have also shown that collagen of osteoarthritic cartilage was more soluble and less resistant to 
proteolytic enzymes, again indicating some change in structure and possibly type of collagen. Disturbances in collagen types have been found in a number of inherited pathological conditions. ${ }^{33}$

In the majority of patients studied here the initiation of symptoms can be explained by previous trauma or a defect in the pars interarticularis, though it is possible that the annular tear patients have been predisposed owing to an inherent defect in the biochemical structure of the disc. It is clear from this study that the affected disc in spondylolisthesis showed a loss in proteoglycan, with the possibility of an accompanying change in collagen types indicated by immunofluorescence. In the annular tear group the intervertebral disc was largely unchanged biochemically, though extended study of 2 patients suggested that localised changes may occur in the region of the posterior fissure.

The mechanisms underlying these secondary biochemical changes remain obscure. There is considerable evidence that chondrocytes can respond to stress and can make some attempt at repair in damaged articular cartilage in rabbits. ${ }^{34}{ }^{35}$ McDevitt and Muir $^{23}$ suggested that chondrocytes synthesised proteoglycans resembling immature cartilage in response to altered mechanical stress, while Caterson and Lowther ${ }^{36}$ have shown a decrease in hexuronic acid content of non-loadbearing cartilage in sheep, together with a decrease in molecular weight of the proteoglycan present. Although in aging and degeneration proteoglycan shows the most obvious quantitative change, there is still speculation as to which, if either, proteoglycan ${ }^{37}$ or collagen ${ }^{38}$ is the first to be affected. Discs from spondylolisthesis patients provide a suitable sample group for biochemical studies, and investigations may show some interesting relationships between biochemical and biochmechanical changes.

We thank the Medical Research Council for financial support for Dr Helen K. Beard.

\section{References}

1 Wiltsie L L. Spondylolisthesis and its treatment. In: Ruge D, Wiltsie L L, eds. Spinal Disorders-Diagnosis and Treatment. Philadelphia: Lea and Febiger, 1977: 193-217.

2 Park W M, McCall I W, O'Brien J P, Webb J K. Fissuring of the posterior annulus fibrosus in the lumbar spine. $B r J$ Radiol 1979; 52: 382-7.

3 McPhee I B, O'Brien J P. Reduction of severe spondylolisthesis. Spine $1979 ; 4: 430-4$.

4 Urban J P G, Holm S, Maroudas A. Diffusion of small solutes into the intervertebral disc. An in vivo study. Biorheology 1978; 15: 203-23.
5 Woessner J F. The determination of hydroxyproline in tissue and protein samples containing small proportions of this amino acid. Arch Biochem Biophys 1961 ; 93: 440-7.

6 Eyre D R, Muir H. Quantitative analysis of types I and II collagens in human intervertebral discs at various ages. Biochim Biophys Acta 1977; 492: 29-42.

7 Bitter T, Muir H. A modified uronic acid carbazole reaction. Anal Biochem $1962 ; 4: 33-4$

8 Stevens R L, Beard H K, Ryvar R, Robertson W R, O'Brien $\mathrm{J}$ P. Biological changes in the anterior annulus fibrosus in patients with low back pain. Submitted for publication.

9 Beard H K, Ryvar R, Brown R, Muir H. Immunochemical localisation of collagen types and proteoglycan in pig intervertebral discs. Immunology in press.

10 Beard H K, Roberts S, O'Brien J P. Immunofluorescent staining for collagen types and proteoglycan in normal and scoliotic human intervertebral discs. J Bone Joint Surg in press.

11 Buckwalter J A, Maynard J A. Cooper R R, Sheathing of collagen fibrils in human intervertebral discs. J Anat 1978; 125: 615-8.

12 Scott J E. Collagen-proteoglycan interaction. Localisation of proteoglycans in tendon by electron microscopy. Biochem J 1980; 187: 887-91.

13 Urban J P G, Holm S, Maroudas A, Nachemson A. Nutrition of the intervertebral disc. An in vivo study of solute transport. Clin Orthop 1977; 129: 101-14.

14 Adams P, Eyre D R, Muir H. Biochemical aspects of development and ageing of human lumbar intervertebral discs. Rheumatol Rehabil 1977; 16: 22-9.

15 Hendry N G C. The hydration of the nucleus pulposus and its relation to intervertebral disc derangment. $J$ Bone Joint Surg 1958; 40B: 132-44.

16 Mitchell P E G, Hendry N G C, Billewicz W Z. The chemical background of intervertebral disc prolapse. $J$ Bone Joint Surg 1961 ; 43B: 141-51.

17 Bushell G R, Ghosh P, Taylor T K F, Sutherland J M. The collagen of intervertebral disc in adolescent idiopathic scoliosis. J Bone Joint Surg 1979; 61B: 501-8.

18 Lipson S J, Muir H. Changes in proteoglycans in experimental intervertebral disc degeneration. Arthritis Rheum in press.

19 Lowther D A, Sandy J D, Sauter V B, Brown H L G. Antigen induced arthritis, decreased proteoglycan content and inhibition of proteoglycan synthesis in articular cartilage. Arthritis Rheum 1978; 21 : 675-80.

20 Venn M, Maroudas A. Chemical composition and swelling of normal and osteoarthrotic femoral head cartilage. Ann Rheum Dis 1977; 36: 121-9.

21 Byers P D, Maroudas A, Oxtop F, Stockwell R A, Venn M F. Histological and biochemical studies on cartilage from osteoarthrotic femoral heads with special reference to surface characteristics. Connect Tissue Res 1977; 5: 41-9.

22 Lippiello L, Hale D, Mankin H J. Collagen synthesis in normal and osteoarthritic human cartilage. $J$ Clin Invest 1977; 59: 593-600.

23 McDevitt C A, Muir H. Biochemical changes in the cartilage of the knee in experimental and natural osteoarthritis in the dog. J Bone Joint Surg 1976; 58B: 94-101.

24 Bollet A J, Nance J L. Biochemical findings in normal and osteoarthritic articular cartilage II. J Clin Invest 1966; 45: 1170-7.

25 Vassan N. Proteoglycan in normal and severely osteoarthritic human cartilage. Biochem J 1980; 187: 781-7.

26 Mankin H J, Lippiello L. Biochemical and metabolic abnormalities in articular cartilage from osteoarthritic human hips. J Bone Joint Surg 1970; 52A : 424-34. 
27 Sylvest J, Hentzer B, Kobayasi T. Ultrastructure of prolapsed disc. Acta Orthop Scand 1977; 48: 32-40.

28 Chrisman O D. Biochemical aspects of degenerative joint disease. Clin Orthop 1969; 64: 77-86.

29 Nimni $M$, Deshmukh $K$. Differences in collagen metabolism between normal and osteoarthritic human articular cartilage. Science $1973 ; 181$ : 751-2.

30 Deshmukh K, Henrick S. Metabolic Changes in the rabbit articular cartilage due to inflammation. Arthritis Rheum 1976; 19: 199-208.

31 Floman Y, Eyre D R, Glimcher M J. Induction of osteoarthrosis in the rabbit knee joint. Clin Orthop 1980; 147: 278-86.

32 Adam M, Musilova J, Deyl Z. Cartilage collagen in osteoarthrosis. Clin Chim Acta 1976; 69: 53-9.

33 Uitto J, Lichenstein J R. Defects in the biochemistry of collagen in diseases of connective tissue. J Invest Dermatol 1976; 66: 59-79.
34 Cheung H S, Cottrell W H, Stephenson K, Nimni M E. In vitro collagen biosynthesis in healing and normal rabbit articular cartilage. J Bone Joint Surg 1978; 60A: 1076-81.

35 Mitchell W, Shepard N. Healing of articular cartilage in intra-articular fractures in rabbits. $J$ Bone Joint Surg 1980 ; 62A : 628-34.

36 Caterson B, Lowther D A. Changes in the metabolism of the proteoglycans from sheep articular cartilage in response to mechanical stress. Biochim Biophys Acta 1978; 540: 412-22.

37 Dingle J T. Recent studies on the control of joint damage: the contribution of the Strangeways Research Laboratory. Ann Rheum Dis 1979; 38: 201-14.

38 Maroudas A. Balance between swelling pressure and collagen tension in normal and degenerate cartilage. Nature 1976; 260: 808-9. 